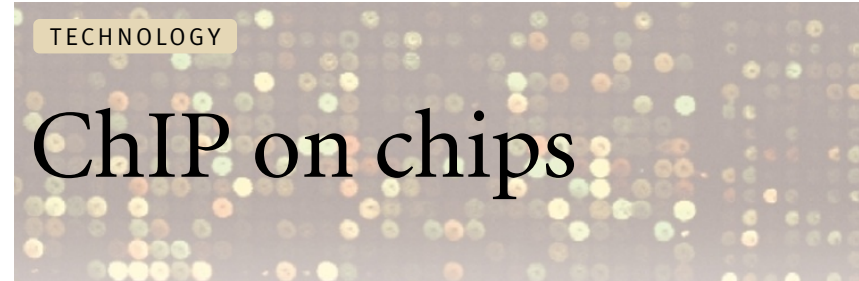

Microarray analysis has become a widely used technique for observing the transcriptional profile of genes at a genome-wide level. But its strength reveals one of its limitations. Observing the patterns of transcriptional activity that occur under different conditions reveals genes that are co-expressed, but cannot distinguish between genes that are under common regulatory control and those whose expression patterns just happen to correlate. What we'd really like to know is, if two genes have matching transcriptional profiles, are they also under the direct transcriptional control of a common upstream regulator? A group led by Richard Young has taken us one step further towards answering this question by developing a microarraybased technique that can monitor the chromosomal locations at which protein-DNA interactions occur across the entire yeast genome. Researchers should now be able to identify all the genes whose transcription is directly affected by any DNA-binding protein. The new method involves combining chromatin immunoprecipitation (also known as ChIP) with microarray analysis. After crosslinking a protein of interest to genomic DNA, the complex is immunoprecipitated. Two pools of DNA fragment - those released from the protein and those from control DNA - are amplified and labelled using different fluorescent dyes. These are then hybridized to a microarray plate that contains all $(6,361)$ intergenic sequences in the Saccharomyces cerevisiae genome. The greater the difference in the fluorescent intensity at any fragment on the array, the stronger the binding of the protein to that fragment.

To prove that the method works, the authors performed a genomewide location analysis to identify the sites bound by Gal4 and Ste12, two well-characterized yeast transcription factors.

Gal4 activates genes required to metabolize galactose, from which yeast cells derive energy when glucose is limiting. Only ten loci were bound by Gal4 (and were also activated in the presence of galactose), seven of which were previously known downstream targets of Gal4. The three new genes, such as PCL10, revealed new information about the coordination of different metabolic pathways. For instance, by preventing glycogen formation, the activation of PCL10 could be required to maximize the energy that is obtained from galactose. In response to mating pheromone, Ste12 induces the activation of over 200 genes, but it is not known how many are affected directly. Ste 12 was found to bind to the upstream regions of 29 pheromoneinduced genes, 11 of which are known to be involved in various steps of the mating process. Again, the unknown genes could plausibly have mating-related functions - in cell morphology or in nuclear fusion, for example.

The ability to identify all the genes whose upstream regulatory regions are bound by certain DNA-binding protein should greatly improve the functional studies of proteins, especially where our understanding of their function has been limited by restricted knowledge of their targets.

Tanita Casci

\section{(2) References and links}

ORIGINAL RESEARCH PAPER Ren, B. et al. Genome-wide location and function of DNA binding proteins. Science 290, 2306-2309 (2000)

WEB SITES Richard Young's lab | Experimental data

EVO-DEVO

\title{
The secret is in the head
}

Amphioxus is a popular creature in evo-devo circles, as it is the closest living relative of vertebrates. This singular little fish-like creature (amphioxus literally means sharp at both ends) has a notochord but, unlike vertebrates, it has no skull and only a simple forerunner of the brain. The fact that amphioxus has no head is central to the transgenic studies reported by Peter Holland's group, who have recently demonstrated that the vertebrate head evolved by tweaking the more rudimentary developmental systems that specify the anterior-most structures in these invertebrates.

Hox genes encode a family of conserved transcription factors that specify the location of cells along the anterior-posterior axis of the embryo. Amphioxus is like the other invertebrates in that it has a single cluster of Hox genes, whereas all vertebrates have three to eight copies of each set.

Now, these authors show that the control regions of amphioxus Hox genes that pattern the anterior-most structures are recognized in vertebrates, where they can drive expression to specific head structures.

Seven cis-regulatory sequences, taken from three amphioxus Hox genes (AmphiHox1-3), were used to drive expression of a reporter gene (lacZ) in mouse and chick embryos. In both model vertebrates, the same four DNA fragments reproduced the expression of native vertebrate Hox genes in three head tissues: the developing hindbrain, the neural crest and the neurogenic placodes - the latter two of which are absent in amphioxus.

In addition, the expression of lac $Z$ was sensitive to the concentration of retinoic acid, which is believed to be one of the morphogens that controls the spatial expression of individual Hox genes along the anterior-posterior axis.
Nature could have devised a whole new genetic network to create a head where previously there was none. Instead, it seems that she has economically crafted evolutionary innovations by manipulating the existing genetic machinery.

We are used to hearing about the conservation of gene-coding regions, but probably less so about the conservation of their control regions, which, by contrast, we tend to think of as engendering change by altering the time and place of gene expression. This work is therefore remarkable in highlighting the strong selection pressures that have preserved genetic networks over the 500 million years that separate amphioxus from its vertebrate (and headendowed) relatives.

Tanita Casci

(1) References and links ORIGINAL RESEARCH PAPER Manzanares, M. et al. Conservation and elaboration of Hox gene regulation during evolution of the vertebrate head. Nature 408, 854-857 (2000) FURTHER READING Finnerty, J. R. Head start. Nature 408, 778-781 (2000) | Ferrier, D. E. K. et al. The amphioxus Hox cluster: deuterostome posterior flexibility and Hox14. Evol. Dev. 2, 284-293 (2000) WEB SITE Peter Holland's lab

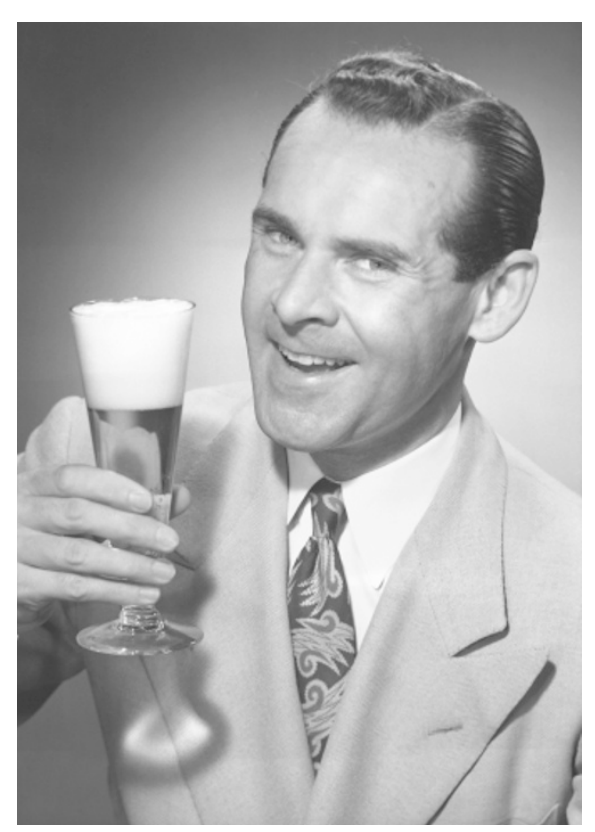

